\title{
A Randomized, Double Blinded, Split-Face Study of the Efficacy of Using a Broad Spectrum Sunscreen with Anti-Inflammatory Agent to Reduce Post Inflammatory Hyperpigmentation After Picosecond
} Laser

\author{
Thanaporn Puaratanaarunkon (D), Pravit Asawanonda (D) \\ Division of Dermatology, Department of Medicine, Faculty of Medicine, Chulalongkorn University, Bangkok, Thailand \\ Correspondence: Pravit Asawanonda, Division of Dermatology, Department of Medicine, Faculty of Medicine, Chulalongkorn University, 1873 Rama \\ IV Road, Pathumwan, Bangkok, I0330, Thailand, Tel/Fax +662-2564253, Email Fibrosis@gmail.com
}

Background: Post inflammatory hyperpigmentation (PIH) is a sequela of laser procedures observed commonly in darker-skin individuals. In general, regular UV filters are beneficial in preventing PIH, but the comparison with sunscreen containing antiinflammatory ingredients remains unexplored.

Objective: To compare the efficacy of a sunscreen with anti-inflammatory agent (sunscreen A) in the reduction of PIH after a picosecond laser with that of regular sunscreen (sunscreen B).

Methods: Fifty-nine acne vulgaris and acne scar patients with skin phototypes III and IV were treated with 1 session of picosecond laser with the microlens array to the whole face. Sunscreens A and B were randomized to be applied on either side of the face. Hyperpigmentation assessed by brown score mode on Visia ${ }^{\circledR}$, acne quantity, porphyrins and patient satisfaction were evaluated at baseline, weeks 1, 2, 4 and 6 .

Results: Sunscreen A caused a higher reduction of the brown score compared to the other side but there was no statistically significant difference. Interestingly, a significant decrease of inflammatory acne lesions compared with baseline was observed as early as week 2 on the sunscreen A side (weeks 2, 4 and 6; $P=0.017, P=<0.001$, and $P=<0.001$, respectively). Compared with sunscreen B, levels of porphyrins on sunscreen A side were significantly less at weeks 1 and 6 (weeks 1 and $6 ; P=0.022$ and $P=0.029$, respectively). Conclusion: This study demonstrated a tendency towards lower post-laser pigmentation when the sunscreen with anti-inflammatory agents was applied. This product also had an effective outcome as an adjunctive treatment option of acne vulgaris.

Thai Clinical Trials Registry ID: TCTR20210305004 (URL: http://www.thaiclinicaltrials.org/show/TCTR20210305004).

Keywords: post inflammatory hyperpigmentation, picosecond laser, sunscreen, acne vulgaris, licochalcone A

\section{Introduction}

Post-inflammatory hyperpigmentation (PIH) results from either endogenous or exogenous etiology, and ironically aesthetic procedures. ${ }^{1}$ Due to higher levels of melanin in the epidermis, individuals with skin of colors are more prone to PIH. ${ }^{2}$ The exact pathogenesis remains unclear but several lines of evidence suggest that inflammatory mediators, cytokines and reactive oxygen species, released during inflammation, can participate in the regulation of melanogenesis. Among these, leukotriene C4 profoundly increases tyrosinase activity, a key enzyme in the melanogenesis. ${ }^{3}$ On top of the epidermal pigmentation, dermal melanosis can also occur when the basal layer is disrupted during the inflammatory process or aesthetic procedures. This often results in more profound and long-lasting grayish brown hyperpigmentation. ${ }^{4} \mathrm{PIH}$ is also associated with adverse psychological effects. ${ }^{5}$ To minimize this, the most crucial step lies in prevention using sunscreen, corticosteroid and epidermal cooling devices. $^{6-9}$ 
Novel UV filters are an integral part in the management of melasma and other pigmentary disorders. Nonetheless, they might not be efficacious in mitigating the inflammatory cytokines and importantly, reactive oxygen species, mentioned above. Several anti-oxidants, anti-inflammatory agents have been added to sunscreens to enhance their protective effects. Licochalcone A, has been shown to have an inflammatory effect and inhibits pro-inflammatory cytokines, which are part of the PIH mechanism. ${ }^{10}$ In addition, 1-carnitine is also added in certain sunscreens to decrease sebum production, which may be beneficial to management of acne vulgaris. Our study aims to evaluate the efficacy of sunscreen A, which contains licochalcone A and l-carnitine in reducing PIH after a picosecond laser treatment among acne vulgaris and atrophic acne scar patients. This non-ablative laser generated an ultra-short pulse duration and high fluence of energy which could improve skin texture, including acne scars. Furthermore, our study also examines the efficacy of sunscreen A by comparing with an otherwise similar UV filter without the two additive ingredients (sunscreen B).

\section{Methodology}

This was a randomized, double blinded, split-face clinical study carried out at King Chulalongkorn Memorial Hospital, Thailand, from March to May 2021. Sixty patients aged above 18 years of skin phototypes III and IV with mild-tomoderate acne vulgaris (The Leeds Revised Acne Grading System) and mild-to-moderate atrophic acne scar (A Qualitative Global Scarring Grading System) were recruited. Exclusion criteria included pregnant or lactating females, irregular menstrual cycles or clinically suspected polycystic ovarian syndrome patients, patients with other pre-existing skin conditions, allergy to product ingredients, patients receiving isotretinoin, corticosteroid or hormonal therapy within the preceding 3 months, patients who underwent laser procedure or phototherapy within the preceding 1 month, and those who used topical treatment for acne vulgaris or oral antibiotics within the preceding 2 weeks.

Enrolled patients washed their faces with a mild cleanser and lidocaine $2.5 \%$ and prilocaine $2.5 \%$ cream (a eutectic mixture of local anesthetic) (AstraZeneca LP, Wilmington, Delaware) was applied to the treatment area under occlusion for one hour. Patients were treated with picosecond laser (Enlighten III TM, Cutera USA), microlens array mode, with the following parameters; spot size $8 \mathrm{~mm}$, fluence $0.8 \mathrm{~J} / \mathrm{cm} 2$, frequency $10 \mathrm{~Hz}, 4500$ shots on both sides of the face. An air-cooling device $\left(\right.$ Koolio $^{\circledR}$, Daolmed Korea) was used at a cooling level 3 during treatment. The laser was operated by the same dermatologist (P.A.). Hydrophilic base was administered to patients as an immediate post laser treatment. All patients were advised to avoid sun exposure after the laser treatment.

Each side of patient's face was randomized to receive sunscreens A or B by block randomization. Sunscreen A was a broad spectrum sunscreen, containing licochalcone A, l-carnitine, octocrylene, butyl methoxy dibenzoylmethane (avobenzone/BMBM), homosalate (Eucerin ${ }^{\circledR}$, Hamburg, Germany) while the other side received sunscreen B with the same UV filters, yet without anti-inflammatory and sebum reduction agents. Both products were dispensed using identical packages with different code labels. On days 0-3, patients were allowed to use only mild cleanser and hydrophilic base twice daily. To avoid irritation, sunscreen and topical treatment for acne vulgaris, according to this protocol, were started from day 4. The patients were instructed to apply both sunscreens in the morning as the last step. The amount of both products should be enough to cover each side of the face. Throughout the study period, other topical products were not permitted.

The primary outcome was the degree of hyperpigmentation as objectively measured by Canfield's RBX ${ }^{\circledR}$ Technology, which relies on the brown score mode from Visia ${ }^{\circledR}$ (Canfield Scientific, Parsippany, NJ, USA). The secondary outcomes were inflammatory and non-inflammatory acne lesion counts as evaluated by two dermatologists, porphyrin counts under UV fluorescence from Visia ${ }^{\circledR}$ and patient satisfaction score (0-10). All measurements were performed at baseline, week 1, week 2, week 4 and week 6. Following each session, patients' skin tolerability, including burning sensation, itching, scale and redness was also assessed.

Statistical analysis was performed by using Stata version 15 (StataCorp). All data were reported as mean and standard deviation (SD) or counts and percentages for continuous and categorical variables, respectively. Two-tailed paired $t$-test was used to compare the outcomes at baseline with those at the end of study. Independent sample $t$-test was used to compare the outcomes between the groups. A p-value less than 0.05 was considered statistically significant.

This study was conducted in accordance with the Declaration of Helsinki and approved by the Institutional Review Board of the Faculty of Medicine, Chulalongkorn University, Thailand (protocol no.045/64). Informed consent was 
Table I Baseline Demographics of Enrolled Patients

\begin{tabular}{|l|c|c|}
\hline Clinical Characteristics (n=Number of Patients) & (n =59) \\
\hline Age (years) & 28.56 & \pm 6.17 \\
$\quad$ Mean (SD) & & \\
Gender, $n$ (\%) & 42 & $(71.2)$ \\
$\quad$ Female & 17 & $(28.8)$ \\
Male & & \\
Skin phototype, $n$ (\%) & 33 & $(55.9)$ \\
Type III & 26 & $(44.1)$ \\
Type IV & & \\
Baseline acne grade, $n$ (\%) & 26 & $(44.1)$ \\
Mild & 33 & $(55.9)$ \\
Moderate & & \\
Baseline acne scar grade, $n$ (\%) & 31 & $(52.5)$ \\
Mild & 28 & $(47.5)$ \\
$\quad$ Moderate & & \\
\hline
\end{tabular}

provided to all participants prior to enroll the study. Additional informed consent was obtained from a subject whose photographs were included in this article. The Thai clinical trials registration number is TCTR20210305004.

\section{Results}

In total, 60 patients were enrolled and underwent a laser procedure. There was one patient who became pregnant after laser treatment and had to withdraw from the study. Baseline patient demographic characteristics are shown in Table 1. The mean age was $28.56 \pm 6.17$ years (range 19-46 years). The majority was female ( $\mathrm{N}=42,71.2 \%)$. Most of the subjects had skin phototype III $(\mathrm{N}=33,55.9 \%)$. At baseline, approximately $55.9 \%$ of patients had moderate acne vulgaris, while $52.5 \%$ had mild acne scar.

Regarding the primary outcome, the sunscreen A side showed a gradual decrease in the scores from $35.69 \pm 5.81$ at baseline to $35.36 \pm 5.98$ at week 6 . Similarly, the mean score of the sunscreen B side declined from $35.83 \pm 5.12$ at baseline to $35.69 \pm 5.42$ at week 6 . However, there was no statistically significant difference in brown score between both sides of the face (Table 2; Figure 1A). After the laser, only one patient displayed clinically-evident PIH on both sides of the face, but the side with the sunscreen A demonstrated less intensity and earlier amelioration (Figures 2 and 3).

Interestingly, in subjects with active acnes, a faster onset of inflammatory acne lesion reduction was observed in the sunscreen A side. A statistically significant reduction from baseline occurred from week 2 until the end of the study (weeks 2, 4 and 6; $P=0.017, P=<0.001$, and $P=<0.001$, respectively), while the other side demonstrated a significant decrease in inflammatory acne count later (week 6, $P=0.026$ ) (Figure $1 \mathrm{C}$ ). In contrast, the non-inflammatory acne

Table 2 Mean Brown Score at Baseline, Week I, Week 2, Week 4 and Week 6 Follow-Up Visit

\begin{tabular}{|c|c|c|c|c|c|c|c|c|c|}
\hline \multirow[t]{2}{*}{ Week } & \multicolumn{4}{|c|}{ Sunscreen A } & \multicolumn{4}{|c|}{ Sunscreen B } & \multirow[t]{2}{*}{$P$-value } \\
\hline & $\begin{array}{l}\text { Mean } \\
\text { Brown } \\
\text { Score }\end{array}$ & SD & $\begin{array}{l}\text { Mean Change } \\
\text { from Baseline }\end{array}$ & $\begin{array}{c}\text { P-value } \\
\text { (Compare to } \\
\text { Baseline) }\end{array}$ & $\begin{array}{l}\text { Mean } \\
\text { Brown } \\
\text { Score }\end{array}$ & SD & $\begin{array}{l}\text { Mean Change } \\
\text { from Baseline }\end{array}$ & $\begin{array}{c}\text { P-value } \\
\text { (Compare to } \\
\text { Baseline) }\end{array}$ & \\
\hline 0 & 35.69 & 5.81 & & & 35.83 & 5.12 & & & 0.886 \\
\hline I & 35.24 & 5.58 & -0.42 & 0.312 & 35.46 & 5.25 & -0.20 & 0.416 & 0.824 \\
\hline 2 & 35.54 & 5.36 & -0.14 & 0.743 & 35.89 & 5.25 & 0.05 & 0.904 & 0.720 \\
\hline 4 & 35.32 & 5.43 & -0.37 & 0.373 & 36.08 & 5.36 & 0.20 & 0.573 & 0.449 \\
\hline 6 & 35.36 & 5.98 & -0.24 & 0.491 & 35.69 & 5.42 & -0.03 & 0.721 & 0.758 \\
\hline
\end{tabular}



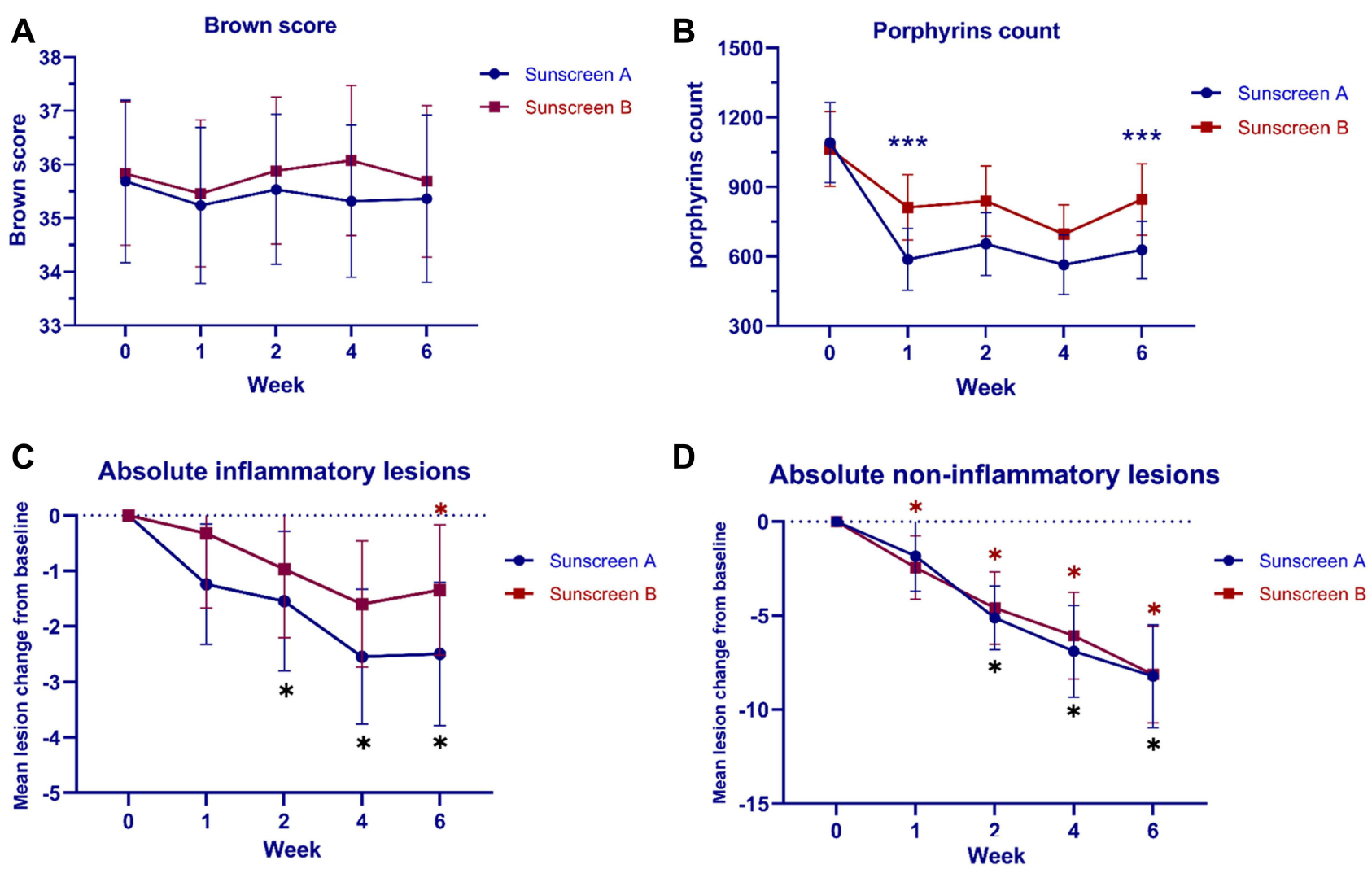

Figure I (A) Mean brown score (B) Mean porphyrins count (C) Mean change of inflammatory acne lesion counts (D) Mean change of non-inflammatory acne lesion counts at baseline, week I, week 2 , week 4 and week 6 follow-up visit. ( $* * * P<0.05$, compared between two products, $* p<0.05$, compared with baseline).

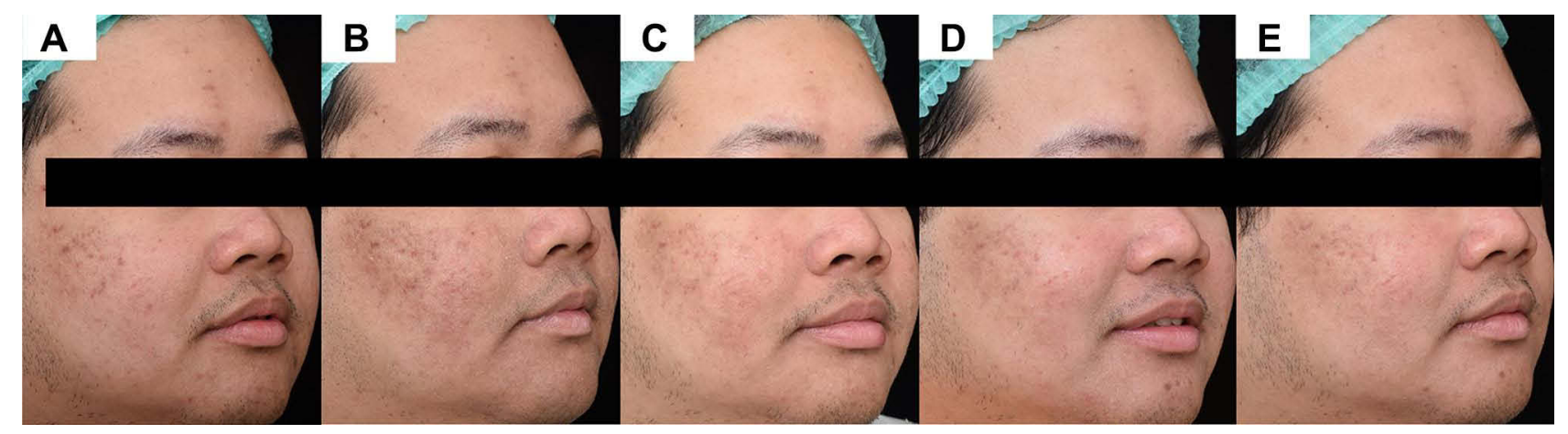

Figure 2 Postoperatively treated side with the sunscreen A. (A) Baseline, (B) I-week I, (C) 2-week, (D) 4-week, and (E) 6-week follow-up visit.

lesions on the sunscreen B side were reduced from baseline with statistical significance since 1-week follow up study (weeks 1, 2, 4 and 6; $P=<0.001$ ), whereas the sunscreen A side achieved the same improvement at week 2 and subsequent study visits (weeks 2, 4 and 6; $P=<0.001$ ) (Figure 1D). However, the inflammatory and non-inflammatory acne counts did not differ significantly between both groups during the course of the study.

Of note, the porphyrin counts declined substantially from baseline as early as week 1 on both sides of the face. Regarding the intergroup comparison, the porphyrin of the side with the sunscreen A was significantly less compared to the side with the sunscreen B at weeks 1 and 6 (weeks 1 and 6; $P=0.022$ and $P=0.029$, respectively) (Figure 1B).

Subjects' satisfaction score on the sunscreen A side was significantly higher than that of the other side throughout the study period (weeks 1, 2, 4 and 6; $P=0.019, P=0.002, P=0.005$ and $P=0.011$, respectively). No serious or major 


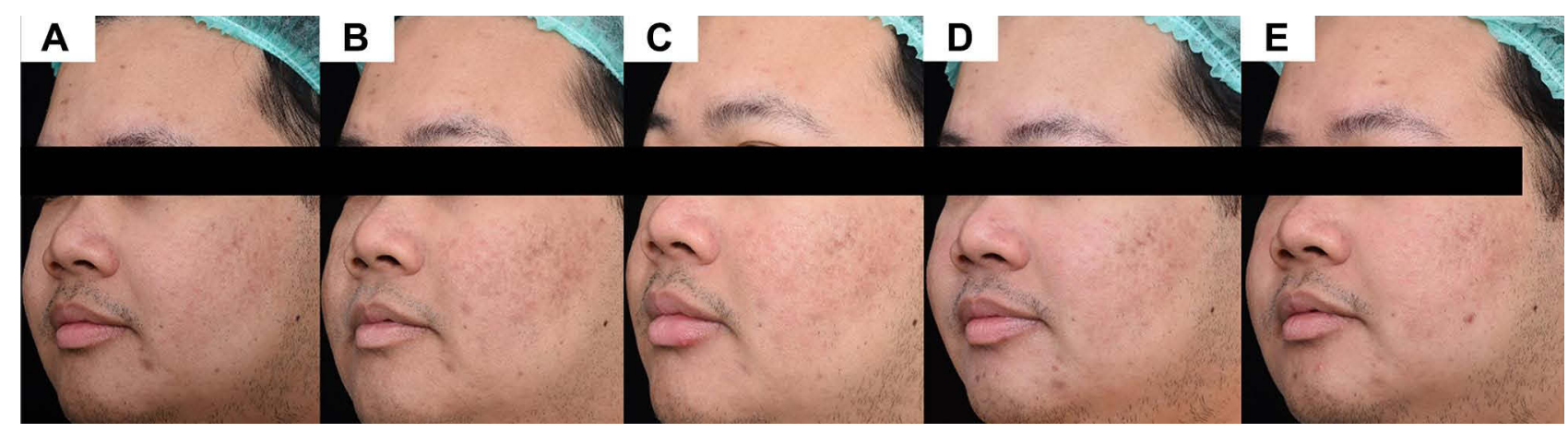

Figure 3 Postoperatively treated side with the sunscreen B. (A) Baseline, (B) I-week I, (C) 2-week, (D) 4-week, and (E) 6-week follow-up visit.

adverse effects were reported. Only some patients were reported to have side-effects while using the sunscreen A, including burning sensation (5.95\%) redness (3.38\%) dryness (1.28\%) and itching (0.43\%). All of the aforementioned symptoms developed only at the beginning of the study and were resolved spontaneously without discontinuation of sunscreen or additional treatment.

\section{Discussion}

PIH can be observed as a common sequela from various laser procedures, especially ablative resurfacing laser. Patients with darker skin types are considerably more prone to developing PIH with greater intensity. Chan et al reported the incidence of PIH subsequent to fractional ablative CO2 laser among Chinese patients with SPTs III and VI, whereby almost $55.5 \%$ of patients developed PIH at 1 month post-treatment. ${ }^{11}$ Therefore, this issue remains a major concern for patients who intend to undergo laser procedures.

According to previous studies, the pathogenesis of PIH was postulated to result from melanogenesis triggered by an inflammatory process. In terms of PIH following laser procedures, thermal damage zone underneath the skin occurs during irradiation and consequently triggers an inflammatory response within the treated site. Eicosanoid derived from arachidonic acid, cytokines and chemokines are subsequently produced and play an influential role in the melanocyte activation, resulting in the elevation of melanin synthesis. In addition, the heat from energy based device also disturbs the epidermis, which precipitates pigment incontinence. ${ }^{7,12,13}$

It is noteworthy that only one patient in our study developed clinically-observable PIH. The difference in laser characteristics was suggested to be responsible for this finding. A short pulse duration of picosecond laser yields less tissue damage to the tissue. ${ }^{14}$ The degree of hyperpigmentation in this subject was obviously higher in the sunscreen $\mathrm{B}$ side. This is arguably due to the anti-oxidant/anti-inflammatory effects of the ingredients in sunscreen A. ${ }^{10,15}$ These agents have also been shown to significantly reduce ROS formation from high energy visible light irradiation. ${ }^{16}$ The antiirritative potency and better photoprotection therefore inhibit the pathogenesis of PIH. This result was in line with the study by Wanitphakdeedecha et al which highlighted the effective outcome of this agent. ${ }^{6}$

With regard to the efficacy of acne therapy, acne lesion counts, and levels of porphyrin produced by Cutibacterium acnes (C. acnes) were used as a tool for evaluation. The sunscreen A side showed a remarkable reduction of porphyrin and a rapid improvement of inflammatory acne lesions at week 2 . From the aforementioned effects, anti-inflammatory ability of licochalcone A was suggested to ameliorate the inflammation in acne vulgaris. Besides, licochalcone A has antibacterial effects against $C$. acnes and NLRP3 inflammasome blockage which helps control skin inflammation induced by $C$. acnes. ${ }^{17}$ Additionally, L-carnitine is involved in beta-oxidation enhancement and decreased intracellular lipid content in sebocytes, bringing about a significant sebum reduction. ${ }^{18}$

The strengths of our study was the split-face design with proper control. The use of clinical and participants' evaluations in conjunction with objective measurements also added values to the assessments. 


\section{Study Limitation}

Limitations include the enrollment of skin types III and IV and only Asian subjects, which limit generalizability. Also, sunscreens were initiated on day 4 after laser treatment, a common practice among many dermatologists. We believe there might be a more prominent difference should the agents be started earlier after laser treatment. This issue warrants further evaluations.

\section{Conclusion}

Taken together, our study suggested that the sunscreen A with anti-inflammatory agents might prevent post-laser PIH, yet further studies are warranted. In acne vulgaris patients, this sunscreen might be beneficial and could be used in combination with other anti-acne preparations for better outcome.

\section{Data Sharing Statement}

The data that support the findings of this study are available on request from the corresponding author, [PA]. The data are not publicly available due to ethical restrictions.

\section{Acknowledgment}

This study was financially supported by Beiersdorf (Thailand) Co., Ltd. The authors are responsible for the accuracy and integrity of the data.

\section{Funding}

Beiersdorf (Thailand) Co., Ltd.

\section{Disclosure}

The authors report no conflicts of interest in this work.

\section{References}

1. Cestari TF, Dantas LP, Boza JC. Acquired hyperpigmentations. An Bras Dermatol. 2014;89:11-25. doi:10.1590/abd1806-4841.20142353

2. Sofen B, Prado G, Emer J. Melasma and post inflammatory hyperpigmentation: management update and expert opinion. Skin Therapy Lett. 2016;21:1-7.

3. Kaufman BP, Aman T, Alexis AF. Postinflammatory hyperpigmentation: epidemiology, clinical presentation, pathogenesis and treatment. Am J Clin Dermatol. 2018;19:489-503. doi:10.1007/s40257-017-0333-6

4. Halder RM, Rodney IJ. Why are there so few effective treatments for pigmentary disorders of the skin? Expert Rev Dermatol. 2012;7:109-112. doi:10.1586/edm.12.9

5. Lacz NL, Vafaie J, Kihiczak NI, Schwartz RA. Postinflammatory hyperpigmentation: a common but troubling condition. Int J Dermatol. 2004;43:362-365. doi:10.1111/j.1365-4632.2004.02267.x

6. Wanitphakdeedecha R, Phuardchantuk R. The use of sunscreen starting on the first day after ablative fractional skin resurfacing. $J$ Eur Acad Dermatol Venereol. 2014;28:1522-1528.

7. Uaboonkul T, Nakakes A, Ayuthaya PK. A randomized control study of the prevention of hyperpigmentation post Q-switched Nd: yAGlaser treatment of Hori nevus using topical fucidic acid plus betamethasone valerate cream versus fucidic acid cream. $J$ Cosmet Laser Ther. 2012;14:145-149. doi:10.3109/14764172.2012.682066

8. Lueangarun S, Tempark T. Efficacy of MAS063DP lotion vs $0.02 \%$ triamcinolone acetonide lotion in improving post-ablative fractional CO(2) laser resurfacing wound healing: a split-face, triple-blinded, randomized, controlled trial. Int J Dermatol. 2018;57:480-487. doi:10.1111/ijd.13922

9. Manuskiatti W, Eimpunth S, Wanitphakdeedecha R. Effect of cold air cooling on the incidence of postinflammatory hyperpigmentation after Q-switched Nd: yAGlaser treatment of acquired bilateral nevus of Ota like macules. Arch Dermatol. 2007;143:1139-1143. doi:10.1001/ archderm.143.9.1139

10. Kolbe L, Immeyer J, Batzer J, et al. Anti-inflammatory efficacy of Licochalcone A: correlation of clinical potency and in vitro effects. Arch Dermatol Res. 2006;298:23-30. doi:10.1007/s00403-006-0654-4

11. Chan NP, Ho SG, Yeung CK, Shek SY, Chan HH. Fractional ablative carbon dioxide laser resurfacing for skin rejuvenation and acne scars in Asians. Lasers Surg Med. 2010;42:615-623. doi:10.1002/1sm.20974

12. Silpa-Archa N, Kohli I, Chaowattanapanit S, Lim HW, Hamzavi I. Postinflammatory hyperpigmentation: a comprehensive overview: epidemiology, pathogenesis, clinical presentation, and noninvasive assessment technique. J Am Acad Dermatol. 2017;77:591-605. doi:10.1016/j.jaad.2017.01.035

13. Davis EC, Callender VD. Postinflammatory hyperpigmentation: a review of the epidemiology, clinical features, and treatment options in skin of color. J Clin Aesthet Dermatol. 2010;3:20-31.

14. Wong T. Picosecond laser treatment for Asian skin pigments: a review. J Cosmet Med. 2019;3:55-63. doi:10.25056/JCM.2019.3.2.55 
15. Barfod L, Kemp K, Hansen M, Kharazmi A. Chalcones from Chinese liquorice inhibit proliferation of T cells and production of cytokines. Int Immunopharmacol. 2002;2:545-555. doi:10.1016/S1567-5769(01)00202-8

16. Mann T, Eggers K, Rippke F, et al. High-energy visible light at ambient doses and intensities induces oxidative stress of skin-Protective effects of the antioxidant and Nrf2 inducer Licochalcone A in vitro and in vivo. Photodermatol Photoimmunol Photomed. 2020;36:135-144. doi:10.1111/ phpp. 12523

17. Yang G, Lee HE, Yeon SH, et al. Licochalcone A attenuates acne symptoms mediated by suppression of NLRP3 inflammasome. Phytother Res. 2018;32:2551-2559. doi:10.1002/ptr.6195

18. Peirano RI, Hamann T, Düsing HJ, et al. Topically applied L-carnitine effectively reduces sebum secretion in human skin. $J$ Cosmet Dermatol. 2012;11:30-36. doi:10.1111/j.1473-2165.2011.00597.x

Clinical, Cosmetic and Investigational Dermatology

\section{Publish your work in this journal}

Clinical, Cosmetic and Investigational Dermatology is an international, peer-reviewed, open access, online journal that focuses on the latest clinical and experimental research in all aspects of skin disease and cosmetic interventions. This journal is indexed on CAS. The manuscript management system is completely online and includes a very quick and fair peer-review system, which is all easy to use. Visit http://www. dovepress.com/testimonials.php to read real quotes from published authors.

Submit your manuscript here: https://www.dovepress.com/clinical-cosmetic-and-investigational-dermatology-journal 